\title{
Gold nanoparticle sucrose complexes as potent anti-inflammatory agents
}

\author{
R. Rahimnia ${ }^{1}$, S.M. Rezayat ${ }^{1}$, R. Torabi ${ }^{2}$, H. Soltanghoraee ${ }^{3}$, \\ B. Mehravi ${ }^{4}$, A. Amani ${ }^{1}$, S. Kharrazi ${ }^{1}$, M. Amanlou ${ }^{5,6}$ \\ ${ }^{1}$ Department of Medical Nanotechnology, School of Advanced \\ Technologies in Medicine, Tehran University of Medical Sciences, Tehran, \\ Iran. \\ ${ }^{2}$ Nanobiomedicine Center of Excellence, Nanoscience and Nanotechnology \\ Research Center, University of Tehran, Tehran, Iran \\ ${ }^{3}$ Reproductive Biotechnology Research Center, Avicenna Research Institute, \\ ACECR, Tehran, Iran \\ ${ }^{4}$ Cellular and Molecular Research Center, Iran University of Medical \\ Sciences, Tehran, Iran. \\ ${ }^{5}$ Department of Medical Biotechnology, School of Advanced Technologies \\ in Medicine, Tehran University of Medical Sciences, Tehran, Iran. \\ ${ }^{6}$ Department of Medicinal Chemistry, Faculty of Pharmacy and Drug Design \\ and Development Research Center, Tehran University of Medical Sciences, \\ Tehran, Iran
}

Corresponding author: M. Amanlou

E-mail: amanlou@tums.ac.ir

Genet. Mol. Res. 17 (4): gmr18037

Received May 29, 2018

Accepted October 02, 2018

Published November 12, 2018

DOI http://dx.doi.org/10.4238/gmr18037

ABSTRACT. Gold nanoparticles (GNPs) with controlled geometric properties are new options for the management of inflammatory responses and therapy for various diseases, including diabetes mellitus, rheumatoid arthritis, and connective tissue diseases. We examined anti-inflammatory impacts of sugar coated GNPs with different sizes on carrageenan-induced rat paw inflammation. The synthesized GNPs were compared with indomethacin, methotrexate and the commercial gold preparation myochrysine. While methotrexate and myochrysine did not have any significant effects on rat paws, GNPs significantly decreased the inflammatory responses, comparable with indomethacin, a standard anti-inflammatory drug in the rat paw model. There was no harmful effect on metabolic activity 
of cells treated with GNPs of different sizes. We conclude that sugar coated GNPs are a promising candidate for new options in the management of inflammatory-related diseases.

Key words: Gold nanoparticles; Carrageenan; Anti-Inflammatory agent; Toxicity

\section{INTRODUCTION}

Gold, the precious metal, has historically been used in Persian traditional medicine for centuries for its therapeutic effects on different diseases, such as sexual disabilities, liver diseases, psychiatric disorders in women, leprosy, conjunctivitis and joint pain (Aghili, 1992; Momen Tonekaboni, 2007). In Eastern medicine, including Chinese medicine, gold and gold-based medications have been used as antimicrobial agents, for management of sexual disabilities and from treatment of pain and bone disorders since several thousand years ago (Gielen and Tiekink, 2005; Vančo et al., 2014). Among the main uses of though gold substrates have been effective in diagnostics and cell photothermolysis treatments, clinical use of gold medications has been little studied in the treatment of rheumatoid arthritis (Burmester and Barthel, 1996; Norn et al., 2011).

Recently, gold salts have been effective in the treatment of inflammatory arthritis and severe rheumatoid arthritis. Most such studies have utilized sodium aurothiomalate (Myochrysine), aurothioglucose (Solganol) and an orally active gold (I) phosphine compound, Auranofin, which can alleviate inflammation responses (Anon). Meanwhile, commercial gold-based anti-arthritis drugs, such as Auranofin and Myochrysine, have been shown to have anti-inflammatory activities, though these pharmaceuticals have some side effects. In recent years, research on medicinal chemistry of gold compounds has focused on development of novel compounds with better performance and/or less negative side effects compared with existing compounds (Vančo et al., 2014).

Gold nanoparticles (GNPs), as an emerging nanomedicine, have received considerable attention because of their ability to inhibit expression of NF-kappa B and their anti-inflammatory activities. These can inhibit expression of various pro-inflammatory mediators and subsequent inflammatory reactions (Zhang et al., 2011). In addition, biocompatibility and easy conjugation with other biomolecules make them ideal candidates for a variety of biological and pharmaceutical applications (Tsai et al., 2007). Tsai et al. (2007) studied pro-inflammatory cytokines including vascular endothelial growth factor (VEGF), tumor necrosis factor alpha (TNF- $\alpha$ ), interleukin-1beta (IL-1 $\beta$ ), interleukin-6 (IL6), which could reduce macrophage infiltration in an arthritis model through GNPs administration (Tsai et al., 2007). Also GNPs have inhibited the secretion of reactive oxygen species (ROS) and lipid peroxidation, by increasing the activity of antioxidant defense enzymes (Jin et al., 2010).

Physical features of GNPs including particle size, shape, surface area, surface chemistry, surface charge, solubility, purity and agglomeration can influence their cytotoxicity and genotoxicity. Synthesized GNPs have a significant tendency to agglomerate or aggregate and in which can control biological responses specifically toxicity properties of nanoparticles. Recently much attention has been paid towards preventing 
GNPs from aggregating. Recent work by Qi et al. has shown that disaccharide cannot reduce $\mathrm{HAuCl}_{4}$ to generate gold nanoparticles (Qi et al., 2004). However, carbohydrate coatings significantly decrease aggregation, which facilitate their application even after freeze-drying (Qi et al., 2004).

We investigated anti-inflammatory and cytotoxic properties of GNPs in a rat model. Two sizes of GNPs 21 and $34 \mathrm{~nm}$ were synthesized with desirable geometrical characteristics via the Turkevich method with slightly modification. Carrageenan used to induce inflammation in rat paw. The inflammation responses and toxicity effects of synthesized GNPs evaluated in vitro and in vivo.

\section{Material and Methods}

\section{Material}

All chemicals used in this study were purchased from Merck Co. (Germany) and Sigma-Aldrich Co. (USA) without any further purification. The RPMI 1640 medium, penicillin-streptomycin mixture, phosphate-buffered saline (PBS, pH: 7.4) and fetal bovine serum (FBS) were purchased from Lonza (Verviers, Belgium). Myochrysine and methotrexate were obtained from a local drugstore. Instant ELISA Kits from IBL (Hamburg, Germany) were used to evaluate production of TNF- $\alpha$, IL-1 $\beta$ and IL-6 by an enzyme linked immunosorbent assay (ELISA) method.

\section{Synthesis of GNPs}

GNPs were prepared according to the Turkevich method with a slight modification (Kimling et al., 2006). Briefly, $4 \mathrm{mM}$ chloroauric acid $\left(\mathrm{HAuCl}_{4} .3 \mathrm{H}_{2} \mathrm{O}, 99.9 \%\right)$ was added in $1000 \mathrm{~mL}$ boiling distilled water and then $0.8 \mathrm{~g}$ tri-sodium citrate was quickly added. The mixture was vigorously stirred for 10 min under boiling conditions. The GNPs were formed by stirring for $20 \mathrm{~min}$ without heating. The mixture had a red wine color, which indicates GNPs formation. The lyophilized GNPs were obtained by freeze-drying.

\section{Preparation of sucrose capped GNPs}

Sucrose capped GNPs were prepared according to above mentioned method (Kimling et al., 2006). Briefly, first, chloroauric acid $\left(\mathrm{HAuCl}_{4} .3 \mathrm{H}_{2} \mathrm{O}\right.$, 99.9\%) solution with final $4 \mathrm{mM}$ concentration was added to $1000 \mathrm{ml}$ boiling distilled water at continuous stirring. In the first method, $0.8 \mathrm{~g}$ of trisodium citrate was quickly added, stirred, and kept boiling for another $10 \mathrm{~min}$. After that $100 \mathrm{~g}$ sucrose was added to a prepared GNPs solution and stirred for an additional $30 \mathrm{~min}$. without heating and aqueous solution turned wine red indicating GNPs formation.

In a second method, the GNPs were prepared as above, but $100 \mathrm{~g}$ of sucrose $(10 \%$; $\mathrm{w} / \mathrm{v}$ ) and $0.8 \mathrm{~g}$ of trisodium citrate were add simultaneously to boiling solution of chloroauric acid and heating continues for $10 \mathrm{~min}$ and finally stirred for an additional 30 min. without heating.

The resulting particle suspensions stocked in a brown bottle at $4{ }^{\circ} \mathrm{C}$. The size of the synthesized GNPs was measured by Dynamic Light Scattering (DLS; 90 plus, Brookhaven 
Instruments Corporation, USA) and Transmission Electron Microscopy (TEM; CEM 902A; Zeiss, Oberkochen, Germany). The size of synthesized GNPs were 32 to 34 and 20 to 21 $\mathrm{nm}$ for these methods, respectively.

\section{Carrageenan-induced hind paw edema}

The carrageenan-induced hind paw edema model was used for the determination of anti-inflammatory activity (Amanlou et al., 2005; Farsam et al., 2000; Hansra et al., 2000). In this study, Wistar rats (about $200 \mathrm{~g}$ ) were maintained according to guidelines for proper conduct of animal experiment of the Tehran University of Medical Science. Before each experiment, the rats fasted for $24 \mathrm{~h}$. The rats with non-significant difference in weight were divided into test and control groups ( $\mathrm{n}=5$ each). Each rat was evaluated once.

The synthesized GNPs $\sim 21$ and $\sim 34 \mathrm{~nm}$ in different concentrations, indomethacin (4 $\mathrm{mg} / \mathrm{kg}$ ), and methotrexate $(40 \mathrm{mg} / \mathrm{kg}$ ), were diluted in $0.1 \%$ DMSO (v/v in distilled water) and administered intraperitoneally (i.p.) 30 min prior to the injection of $100 \mu \mathrm{L}$ of $2 \%$ (w/v) carrageenan solution into the plantar side of right hind paws of the rats. The dosages of the GNPs were adjusted to the same level of $\mathrm{Au}$ in a $0.7 \mathrm{mg} / \mathrm{kg}$ dose of myochrysine, which is an anti-inflammatory dose in rheumatoid arthritis. Paw thickness was measured with a digital caliper immediately after the carrageenan injection, which considered as the baseline value for the hind paw thickness. The degree of swelling was evaluated through changes in thickness of the right hind paw. Each of indomethacin and methotrexate (suspended in $0.1 \%$ DMSO individually) were used as positive controls and $0.1 \%$ DMSO (v/v; vehicle) as negative control, respectively. After $6 \mathrm{~h}$, the animals were sacrificed and the kidney and liver were extracted, fixed and stained with a standard hematoxylin/eosin (HE) staining method for cytological evaluation of inflammatory cells infiltration and cellular damage. Blood samples of the distal tail vein were collected for determination of inflammatory markers, including TNF- $\alpha$, IL- $1 \beta$ and IL-6.

\section{GNPs uptake measured by ICP-MS}

To investigate hepatocellular uptake of the substrates in rat liver, liver samples were collected $6 \mathrm{~h}$ after injection of carrageenan in rat paw following sacrificing the animals by cervical dislocation. Samples (1 g of liver tissue) individually were digested in $20 \mathrm{~mL}$ of aqua regia (one-part concentrated $\mathrm{HNO}_{3}$ to three-part concentrated $\mathrm{HCl}$ ) (Coulter et al., 2012). Gold concentration was analyzed by using Induced Coupled Plasma-Mass Spectrometry (ICP-MS, ELAN 6100 PerkinElmer Inc., Waltham, MA, USA) after filtration, which measures the absolute quantity of elemental gold with parts per billion (ppb) sensitivity.

\section{In vitro cytotoxicity test}

In vitro cytotoxic activity was determined by tetrazolium reduction assay (3-[4,5dimethylthiazol-2-yl]-2,5 diphenyl tetrazolium bromide, MTT) in a Human Dermal Fibroblast (HDF-1) cell line, which was donated by the National Center for Genetic and Biological Reserves in Iran (Mehravi et al., 2013). The cells were cultured in Dulbecco's Modified Eagle Medium supplemented with 10\% (w/v) fetal bovine serum and 1\% (v/v) 
penicillin-streptomycin. The medium was exchanged for a serum-free medium after $24 \mathrm{~h}$ incubation and the culture media was stabilized for an additional $24 \mathrm{~h}$ of incubation.

In the MTT assay, the HDF- 1 cell line $\left(1 \times 10^{4}\right.$ cells per well $)$ was incubated with five different concentration of synthesized GNPs $(100 \mu \mathrm{g} / \mathrm{mL}, 500 \mu \mathrm{g} / \mathrm{mL}, 1,5$ and 10 $\mathrm{mg} / \mathrm{mL}$ ), in a 96 well tissue culture plate for $48 \mathrm{~h}$. The untreated cells were used as a control condition. Then, cells were washed with PBS before adding MTT solution $(20 \mu \mathrm{L}$ of 5 $\mathrm{mg} / \mathrm{mL}$ MTT solution per each well). After $4 \mathrm{~h}$, the absorbance of the developed color of each well was measured at $570 \mathrm{~nm}$ using a microplate reader (ELx800 Absorbance Microplate Reader, BioTek, Winooski, USA) (Mosayebnia et al., 2014), (Tabasi et al., 2013), (Mahernia et al., 2017).

\section{Evaluation of cytokine secretion}

As a model of in vitro inflammatory response, the expression of pro-inflammatory cytokine TNF- $\alpha$, IL- 6 and IL- $1 \beta$ were determined $6 \mathrm{~h}$ after inflammation induction by carrageenan injection. The concentrations of TNF- $\alpha$, IL- 6 and IL- $1 \beta$ were determined by the Instant ELISA Kit according to the manufactures' manual by Noor Clinical Laboratory.

\section{Statistical analysis}

The results obtained from in vivo and in vitro assays were presented as means \pm standard error of the mean (S.E.M). The significance of the differences between above mentioned test results were assessed by ANOVA analysis with $P<0.05$ considered to be significant (SPSS software, Ver. 20), The differences between hind paw thickness, in vitro cytotoxic, GNPs uptake and cytokine secretion in each individual groups during antiinflammatory testing period were evaluated using a one-way ANOVA test, followed by Tukey's post-hoc test for multiple comparisons. GraphPad Prism 5.02 (GraphPad Software Inc., San Diego, CA, USA) was used to perform the analysis.

\section{Ethic statement}

This study was carried out in accordance with the recommendations in the Guide for the Care and Use of Laboratory Animals of Tehran University of Medical Sciences. The study protocol was approved by the Ethic Committee of experimental studies at the Tehran University of Medical Sciences (Permit Number: 73-2013). The animal tissues for ex vivo experiments were taken post mortem, immediately after the animals were sacrificed by cervical dislocation.

\section{Results and Discussion}

\section{Nanoparticles characterization}

The size of introduced particles in biological systems is one of the most important characteristics for its deposition pattern and fate. Particle size controls feasibility of its accessibility in target tissues, the mode of cellular uptake, and efficiency of particle processing in the endocytic pathway. The size of pure GNPs before sucrose addition was 16 
$\mathrm{nm}$, with a narrow size distribution (Figure 1A). Meanwhile, as shown in Figure 1B, the size of particles was increased to $34 \mathrm{~nm}$ in the synthesis of GNPs by simultaneous adding sucrose to the GNPs suspension. The size of the particles was $21 \mathrm{~nm}$ after adding sucrose in the preparation of the gold nanoparticles (Figure 1C). It seems that adding sucrose simultaneously with tri-sodium citrate covered the surface of GNPs more efficiently in comparison with adding sucrose after preparing nanoparticle method. Moreover, the size of the particles did not change in the freeze-drying process (Figure 1E and F).

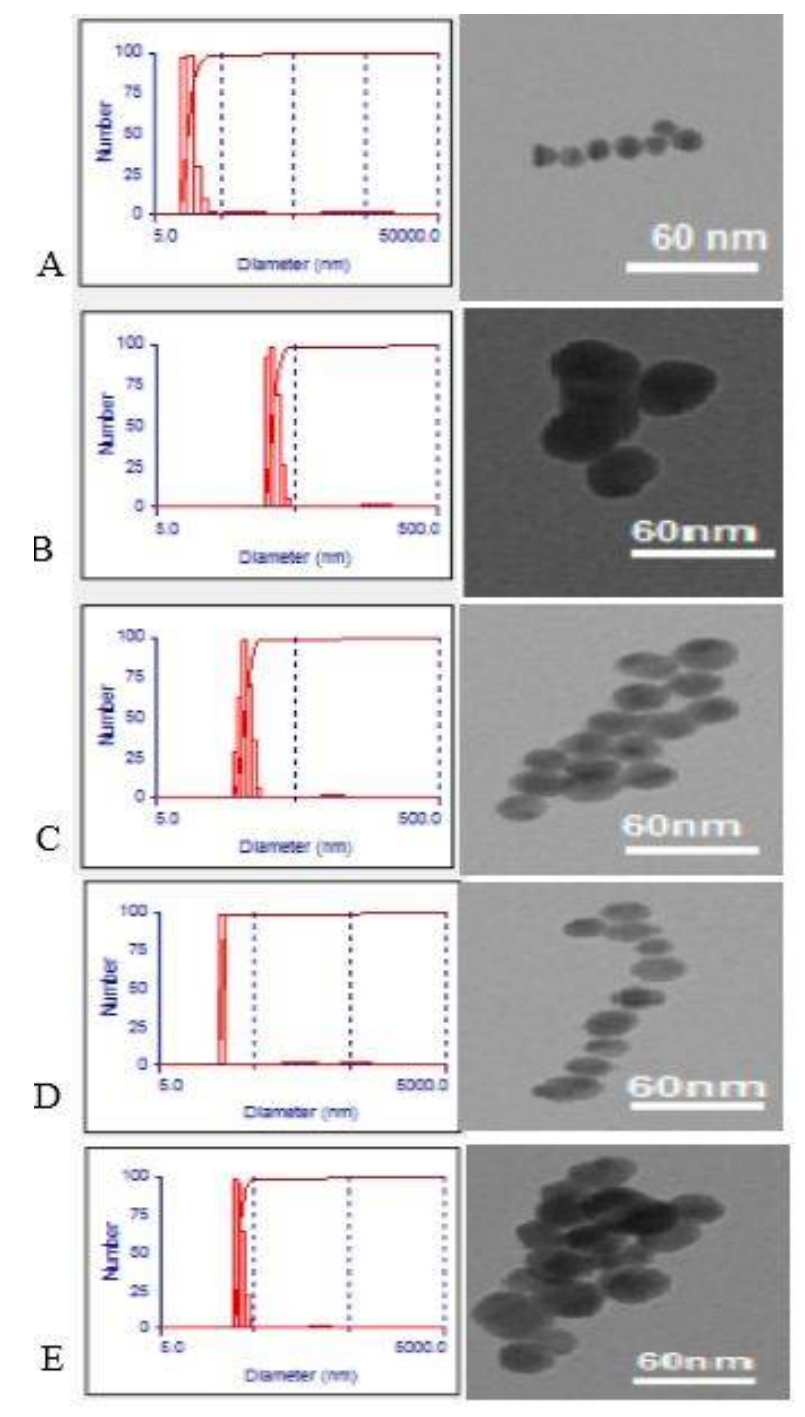

Figure 1. Size characterization of nanoparticles by Dynamic Light Scattering (DLS) and Transmission Electron Microscopy (TEM): (A) pure gold nanoparticles before adding sucrose were about $16 \mathrm{~nm}$ in size, (B) simultaneously added sucrose generated nanoparticles of about $34 \mathrm{~nm},(\mathrm{C})$ adding sucrose after preparation of gold nanoparticles with about $21 \mathrm{~nm}$, (D) $21 \mathrm{~nm}$ GNPs after freeze-drying, and (E) $34 \mathrm{~nm}$ GNPs after freezedrying. 


\section{Anti-inflammatory activity of GNPs}

Anti-inflammatory activity of GNPs reduced the amount of swelling induced when carrageenan-induced edema in the hind paw of rats. Carrageenan, a high-molecular-weight sulfated polysaccharide, has been wildly used for induction of inflammation in animal models in experimental studies (Farsam et al., 2000), (Amanlou et al., 2005), (Hansra et al., 2000) . As the exact causes of inflammation are not yet well known, treatments of this disease have focused on relieving symptoms and reducing progression of inflammation rather than on curing the disease itself (Egan et al., 2002; Hansra et al., 2000; Radhakrishnan et al., 2003). As shown in Figure 2, swelling size was evaluated with a digital caliper. Meanwhile, the myochrysine did not decrease paw edema in rats. The MTX had a minor impact on the degree of swelling; it reduced swelling by $7 \%$ compared with the control. Indomethacin treatment significantly decreased paw edema; the size of swelling was reduced about 32\%. The GNPs at the sizes of 21 and $34 \mathrm{~nm}$ significantly decreased the paw edema of rats by 46 and $37 \%$, respectively, which proved the efficiency of the synthesized GNPs.

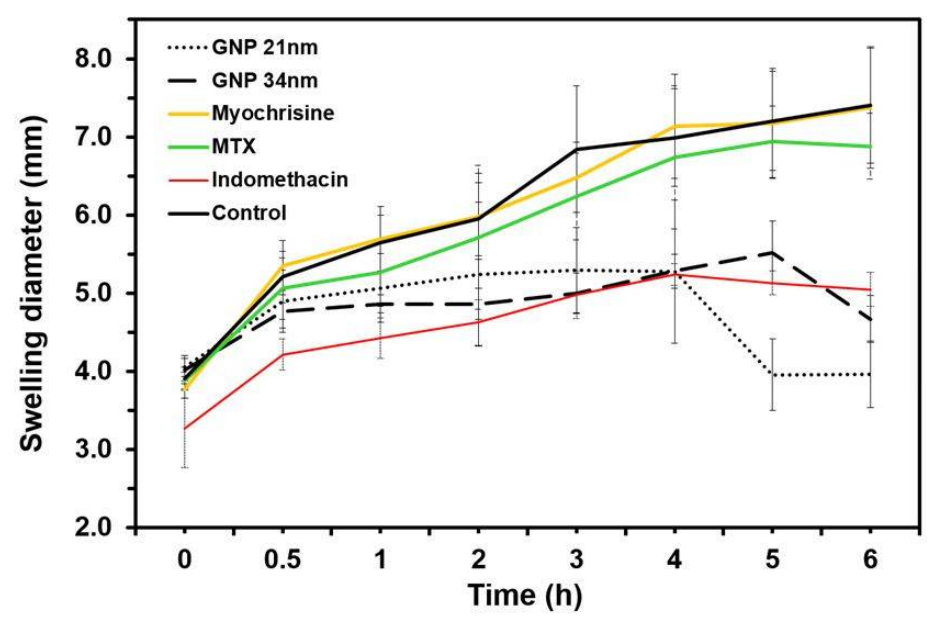

Figure 2. Evaluation of anti-inflammatory activity of gold nanoparticles by reducing the amount of swelling induced by carrageenan in rat paws, measured with a digital caliper.

\section{In vivo distribution and liver GNPs uptake by ICP-MS}

The ICP-MS analysis was performed to evaluate the distribution of GNPs in rat liver $6.5 \mathrm{~h}$ after administration of GNPs. The gold concentration in the liver after administration of $34 \mathrm{~nm}$ GNPs was $70 \pm 11.2 \mathrm{ng} / \mathrm{g}$ of liver tissue. In the case of $21 \mathrm{~nm}$ GNPs, it was $535 \pm 39.8 \mathrm{ng} / \mathrm{g}$ of liver tissue, which demonstrates size dependency of the effectiveness of nanoparticles. The amount of Au was $132 \pm 23.4 \mathrm{ng} / \mathrm{g}$ of liver tissue in myochrysine i.p. injection. As expected, the largest amounts of gold accumulated in the liver after administration of $21 \mathrm{~nm}$ particles. 
The possible toxicity of introduced nanoparticles in biological system has been related to their high surface area per unit mass as well as large number of atoms available for chemical reactions (Boraschi et al., 2017; Jurj et al., 2017; Singh, 2017).

The ICP-MS in vivo results revealed high uptake of GNPs by hepatocytes, the main cells in the liver (>70\%). The uptake of $21 \mathrm{~nm}$ GNPs by liver cells was higher than that by $34 \mathrm{~nm}$ particles. This could be due to the smaller size of nanoparticle or high phagocytic action of hepatic cells, which induced liver toxicity. The ICP-MS results indicated that as the size of GNPs increased, the nanoparticle concentration was decreased (Figure 3). Analysis by one-way ANOVA with $\mathrm{p} \leq 0.01$ revealed that there are significant differences in GNPs uptake among different sizes of GNPs. The uptake of optimal nanoparticle concentration is therefore essential as more nanoparticles mean more toxicity to the human body. Based on these observations and our uptake study results, a higher amount of $21 \mathrm{~nm}$ GNPs was taken up by the liver cells. Consequently, $34 \mathrm{~nm}$ GNPs are more desirable for future clinical studies.

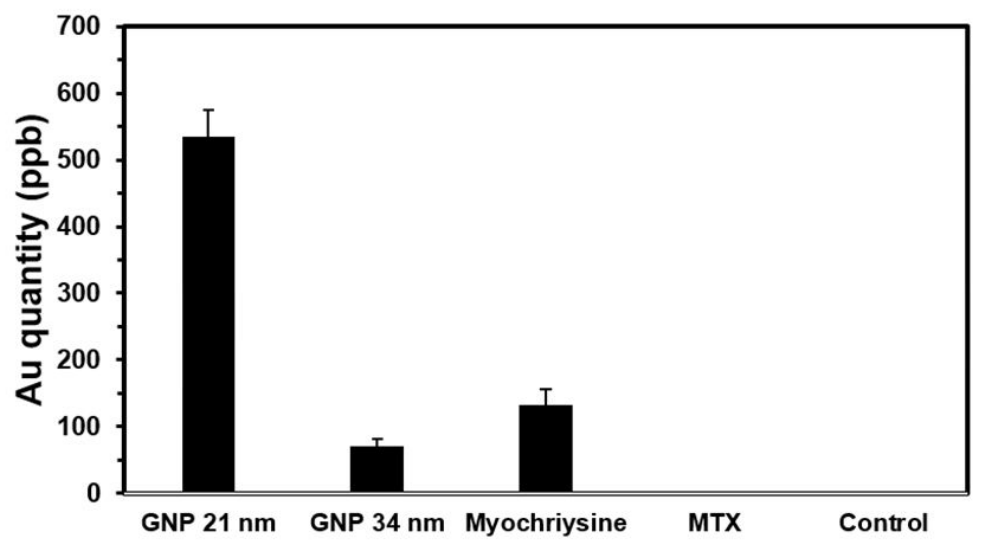

Figure 3. The results of distribution of GNPs measured at $6.5 \mathrm{~h}$ after the injection of $0.7 \mathrm{mg} \mathrm{Au} / \mathrm{kg}$ body weight of rat determined by ICP-MS in liver samples.

\section{In vitro cytotoxicity}

An MTT assay was performed to determine the viability and the rate of HDF-1 cell proliferation on GNPs at $48 \mathrm{~h}$ of culture. The MTT results after $24 \mathrm{~h}$ showed that HDF-1 cells were viable in the presence of $\sim 21$ and $\sim 34 \mathrm{~nm}$ GNPs, which confirms the biocompatibility of these GNPs (Figure 4). The GNPs indicated the increased cell proliferation and cell viability rate of HDF-1 cells during the culture period. In the case of $\sim 34 \mathrm{~nm}$ GNPs, the assay showed higher cell proliferation and viability compared with other cell cultures (TCP and $\sim 21 \mathrm{~nm}$ GNPs). In addition, MTT results showed lower concentrations of $\sim 21 \mathrm{~nm}$ GNPs; the rate of cell proliferation increased and reached a maximum level at lower than $1 \mathrm{mg} / \mathrm{mL}$. Meanwhile, when $\sim 34 \mathrm{~nm}$ GNPs were used, the cell proliferation rate was dose-independent manner and statistically there were no differences in concentrations from $100 \mu \mathrm{g} / \mathrm{mL}$ to $10 \mathrm{mg} / \mathrm{mL}$. These results demonstrate the cytocompatibility of the synthesized GNPs. 

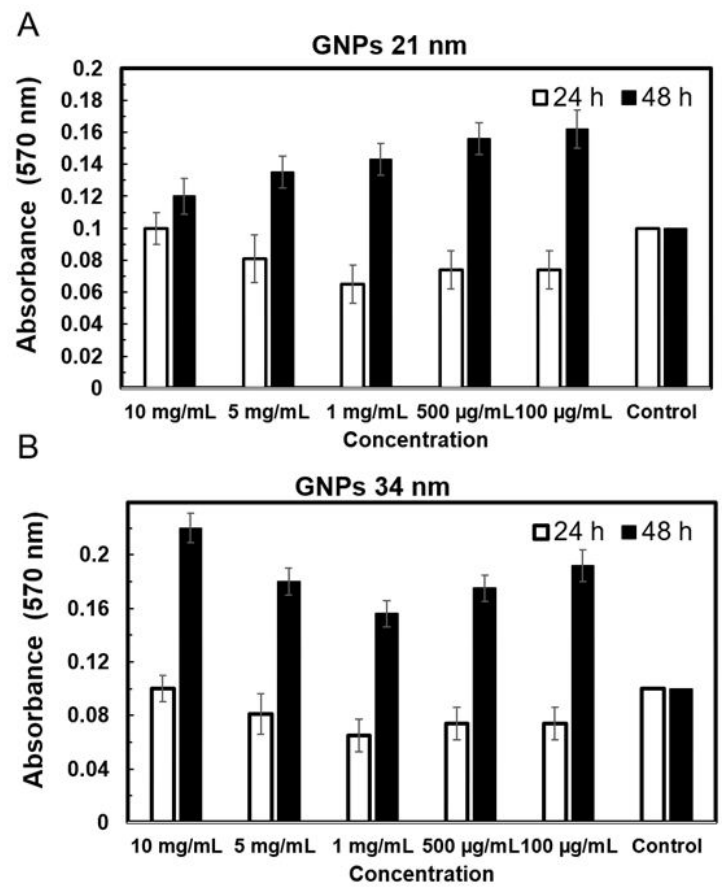

Figure 4. MTT assay results to identify the cytotoxic effects of synthesized GNPs on the proliferation of cells at 24 and 48 h. (A) $21 \mathrm{~nm}$ GNPs and (B) $\sim 34 \mathrm{~nm}$ GNPs.

\section{Effects of GNPs on cytokine secretion}

The effects of the GNPs on the level of pro-inflammatory cytokines, including TNF- $\alpha$, IL-1 $\beta$ and IL-6, were investigated. Both 21 and $34 \mathrm{~nm}$ GNPs did not alter the production of TNF- $\alpha$, IL- $1 \beta$ and IL- 6 compared with control group 6 hours after the injection of carrageenan. The reason for not finding changes in the level of cytokines could be in part attributed to the measurement of cytokines in the plasma of the animal, as it take longer to observe significant alterations in the blood level.

\section{Histopathological findings}

Findings were analyzed by a semi-quantitative method. No glomerulonephritis was seen after application of synthesized GNPs. Mild infiltration of inflammatory cells and mild cellular damage seen as intracellular vacuoles were found in the $21 \mathrm{~nm}$ GNPs treatment (Figure 5A). There was no evidence of severe inflammation among other experimental conditions. Moreover, there was increase in cellular damage in the liver among the experimental conditions. Hepatitis was mild in the $\sim 21 \mathrm{~nm}$ GNPs treatment. Degrees of inflammatory cell infiltration and capsular inflammation (as a sign of peritoneal irritation due to foreign body reaction) in all samples were seen (Figure $5 \mathrm{~B}$ and C). These were mild in the treatment with $34 \mathrm{~nm}$ GNPs and moderate in the $21 \mathrm{~nm}$ GNPs group. 

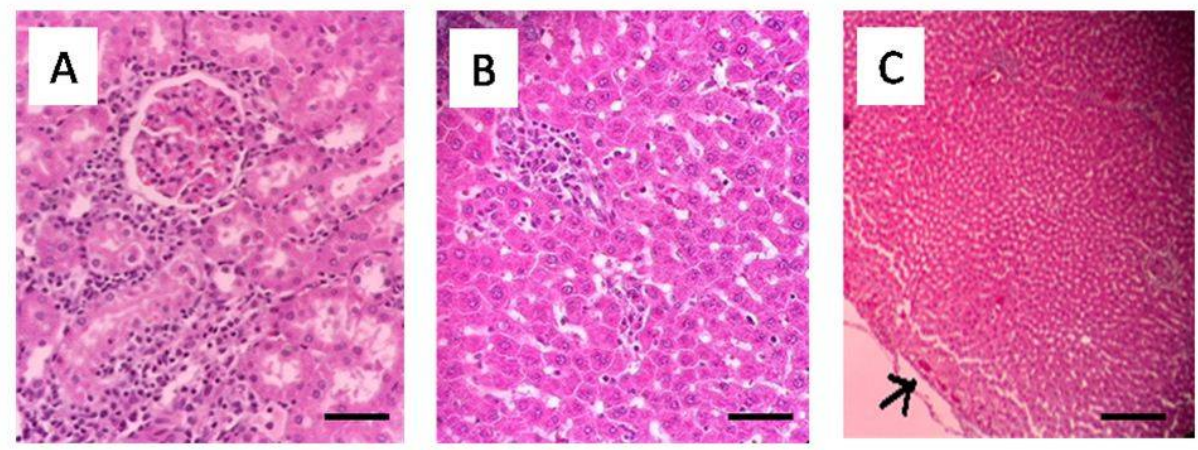

Figure 5. (A) Inflammatory cell infiltration and proximal tubule vacuolization in the kidney in the $21 \mathrm{~nm}$ GNPs group, (B) Parenchymal and portal inflammatory cell infiltration in liver (C) Capsular inflammation in liver. Horizontal bars: $100 \mu \mathrm{m}$.

\section{CONCLUSIONS}

Synthesis, characterization and anti-inflammatory activity of GNPs complexes with sucrose using carrageenan induced rat paw inflammation method is reported. The complexes were thoroughly characterized and their cytotoxicity (in vitro), distribution (in vivo) and anti-inflammatory (in vivo) activities were evaluated. The complexes showed almost no cytotoxicity against HDF-1. The results of in vitro and in vivo anti-inflammatory activity screening indicated that GNPs with average size of 21 and $34 \mathrm{~nm}$ had significant anti-inflammatory effects, comparable with the commercially available drug indomethacin, with minimal histopathological cytotoxicity. We conclude, based on the overall positive findings in the biological testing, that GNPs complexes with sucrose could become usable alternatives to myochrsine or similar preparations as anti-inflammatory agents.

\section{ACKNOWLEDGMENTS}

\section{Council.}

This work was supported by the Tehran University of Medical Sciences Research

\section{REFERENCES}

Aghili, M. (1992) Makhzan Al-Advieh, Tehran, Iran: Secretariat of the Council of the Cultural Revolution.

Amanlou, M, Dadkhah F, Salehnia A, Farsam H, et al. (2005). An anti-inflammatory and anti-nociceptive effects of hydroalcoholic extract of Satureja khuzistanica Jamzad extract. J. Pharm. Pharm. Sci. 8: 102-106.

Anon. Gold Compounds (Systemic). Available at: https://www.drugs.com/mmx/gold-compounds.html.

Boraschi, D, Italiani P, Palomba R, Decuzzi P, et al. (2017). Nanoparticles and innate immunity: new perspectives on host defence. Semin. Immunol. 34: 33-51.

Burmester, GR and Barthel HR. (1996). Effects of gold on the immune system in the treatment of rheumatoid arthritis. $Z$. Rheumatol. 55: 299-306.

Coulter, JA, Jain S, Butterworth KT, Taggart LE, et al. (2012). Cell type-dependent uptake, localization, and cytotoxicity of $1.9 \mathrm{~nm}$ gold nanoparticles. Int. J. Nanomedicine. 7: 2673-2685.

Egan, CG, Lockhart JC, Ferrell WR, Day SM, et al. (2002). Pathophysiological basis of acute inflammatory hyperaemia in the rat knee: roles of cyclo-oxygenase-1 and -2. J. Physiol. 539: 579-587. 
Farsam, H, Amanlou M, Reza Dehpour A and Jahaniani F. (2000). Anti-inflammatory and analgesic activity of Biebersteinia multifida DC. root extract. J. Ethnopharmacol. 71: 443-447.

Gielen, M and Tiekink ERT. (2005) Metallotherapeutic Drugs and Metal-Based Diagnostic Agents: The Use of Metals in Medicine: John Wiley and Sons.

Hansra, P, Moran EL, Fornasier VL and Bogoch ER. (2000). Carrageenan-induced arthritis in the rat. Inflammation 24: 141-155.

Jin, SE, Bae JW and Hong S. (2010). Multiscale observation of biological interactions of nanocarriers: from nano to macro. Microsc. Res. Tech. 73: 813-823.

Jurj, A, Braicu C, Pop LA, Tomuleasa C, et al. (2017). The new era of nanotechnology, an alternative to change cancer treatment. Drug Des. Devel. Ther. 11: 2871-2890.

Kimling, J, Maier M, Okenve B, Kotaidis V, et al. (2006). Turkevich method for gold nanoparticle synthesis revisited. $J$. Phys. Chem. B. 110: 15700-15707.

Mahernia, S, Hassanzadeh M, Sharifi N, Mehravi B, et al. (2017). Structure-based pharmacophore design and virtual screening for novel potential inhibitors of epidermal growth factor receptor as an approach to breast cancer chemotherapy. Mol. Divers.

Mehravi, B, Ahmadi M, Amanlou M, Mostaar A, et al. (2013). Cellular uptake and imaging studies of glycosylated silica nanoprobe (GSN) in human colon adenocarcinoma (HT 29 cell line). Int. J. Nanomedicine. 8: 3209-3216.

Momen Tonekaboni, SM. (2007) Tohfa Tul Momineen, Tehran, Iran: Shahr Publications.

Mosayebnia, M, Shafiee-Ardestani M, Pasalar P, Mashayekhi M, et al. (2014). Diethylentriaminepenta acetic acid glucose conjugates as a cell permeable iron chelator. J. Pharmacol. Pharmacother. 5: 27-32.

Norn, S, Permin H, Kruse PR and Kruse E. (2011). [History of gold--with danish contribution to tuberculosis and rheumatoid arthritis]. Dan. Medicinhist. Arbog. 39: 59-80.

Qi, ZM, Zhou HS, Matsuda N, Honma I, et al. (2004). Characterization of gold nanoparticles synthesized using sucrose by seeding formation in the solid phase and seeding growth in aqueous solution. J. Phys. Chem. B. 108: 7006-7011.

Radhakrishnan, R, Moore SA and Sluka KA. (2003). Unilateral carrageenan injection into muscle or joint induces chronic bilateral hyperalgesia in rats. Pain 104: 567-577.

Singh, AK. (2017). Comparative therapeutic effects of plant-extract synthesized and traditionally synthesized gold nanoparticles on alcohol-induced inflammatory activity in sh-sy5y cells in vitro. Biomedicines 15: 5(4), pii: E70. doi: 10.3390/biomedicines5040070.

Tabasi, MA, Amanlou M, Siadat SD, Nourmohammadi Z, et al. (2013). Novel molecular anti-colorectal cancer conjugate: Chlorambucil-adipic acid dihydrazide-glutamine. Anticancer Agents Med. Chem. 13: 1449-1459.

Tsai, CY, Shiau AL, Chen SY, Chen YH, et al. (2007). Amelioration of collagen-induced arthritis in rats by nanogold. Arthritis Rheum. 56: 544-554.

Vančo, J, Gálikova J, Hošek J, Dvořák Z, et al. (2014). Gold(I) complexes of 9-deazahypoxanthine as selective antitumor and anti-inflammatory agents. PLoS One 9.

Zhang, Q, Hitchins VM, Schrand AM, Hussain SM, et al. (2011). Uptake of gold nanoparticles in murine macrophage cells without cytotoxicity or production of pro-inflammatory mediators. Nanotoxicology 5: 284-295. 